\title{
Strafbegriff, Staatsverständnis und Prozessstruktur
}

\author{
Hans-Heiner Kühne
}

Rezension zu Volker Haas, Strafbegriff, Staatsverständnis und Prozessstruktur, Tübingen (Mohr Siebeck) 2008, 508 S., gebunden 109,- €, ISBN 978-3-16-149402-4

Das Vorhaben des Autors ist ambitioniert, er will eine Neubetrachtung des geltenden Strafverfahrensrechts auf der Basis prozesstheoretischer und „genuin rechtsdogmatischer" Grundlagen unternehmen, um dabei herauszufinden, ob das Strafverfahrensrecht überhaupt noch in sich konsistent und kohärent ist. Er will damit einen Beitrag zur in der Tat längst überfälligen Prozesstheorie des Strafverfahrens leisten, der bewusst zunächst einmal von rechtspolitischen und rechtspragmatischen Überlegungen Abstand nimmt. Dabei scheint ihn vor Allem der unreflektierte Übergang vom Ermittlungs- in ein Absprache- oder Parteimodell - zur Zeit des Abschlusses der Arbeit lag das Verständigungsgesetz noch in weiter Ferne - besondere Sorgen zu bereiten.

Ein großes und notwendiges Thema, welches in seiner Dimension freilich selbst im Rahmen einer Habilitation übermächtig erscheint. Dies insbesondere, weil der Verf. durchaus zurecht betont, dass Staatsverständnis und Strafbegriff durchaus Basisideologien darstellen können, die prägend für das Strafverfahrensrecht sind.

Wenn dann der Autor allerdings seine Überlegungen auf die Positionen von Staatsanwaltschaft und Gericht beschränkt und die Perspektiven von Verteidigung und Opfer ausklammert, dann fragt man sich sogleich, wie das denn möglich sein kann, da doch zumindest die Position der Verteidigung so sehr mit der von Staatsanwaltschaft und Gericht verknüpft ist, dass eine Betrachtung von nur zwei der drei zentralen prozessualen Akteure von vornherein als unvollständig im Rahmen einer theoretischen Gesamtbetrachtung der strafprozessualen Strukturen erscheinen muss. Hierauf wird zurück zu kommen sein.

Machen wir uns aber mit dem Autor auf den Weg, um seinen Gedanken zu folgen und sie - soweit erforderlich - auch kritisch zu hinterfragen.

Zunächst einmal stellt Verf. die gegenwärtige Ausgestaltung des deutschen Strafverfahrens als Anklageprozess mit Ermittlungsgrundsatz dar. Die Beschreibung der Funktion des Gerichts ist vergleichsweise knapp, wohingegen die Position der Staatsanwaltschaft gut historisch unterfüttert und durchaus kritisch als Institution im Niemandsland zwischen Exekutive und Judikative beschrieben wird. Ob allerdings die Strafverfolgungsfunktion der Staatsanwaltschaft mit der Verlesung der Anklageschrift endet, ist selbst bei rein theoretischer Betrachtung zumindest zweifelhaft an-

* Prof. Dr. Dr. h.c. mult. Hans-Heiner Kühne ist emeritierter ordentlicher Professor für Deutsches, Europäisches und Internationales Strafrecht und Strafprozessrecht, Kriminologie an der Universität Trier. 
gesichts der der Staatsanwaltschaft zugewiesenen Rolle, die eingebrachten Beweise einer kritischen Prüfung zu unterziehen und auch nach vorherrschender Ansicht selbst nach Anklageverlesung noch weitere Beweise in die Hauptverhandlung einzubringen. Unstreitig ist hingegen, dass trotz der seit dem 19. Jahrhundert gebräuchlichen Bezeichnung von Staatsanwaltschaft und Beschuldigtem als „Parteien“ keineswegs der Strafprozess als kontradiktorischer beschrieben werden sollte.

Aus dem - wie der Verf. richtig erkennt - heute kaum mehr bedeutsamen Begriff des staatlichen Strafanspruchs will Verf. dann Gründe für eine möglicherweise erforderliche Umgestaltung des Strafverfahrens in einen Parteiprozess folgern.

Auf über hundert Seiten widmet er sich dann der Frage des Einflusses des Staatsverständnisses auf die Einrichtung von Staatsanwaltschaft und Gericht. Dabei erscheinen die ersten 30 Seiten, bei denen es ausschließlich um den Zivilprozess geht, als etwas breit Angesichts der begrenzten Bedeutung für die im Anschluss behandelten Fragen des Strafverfahrensrechts. Bei letzterem zeichnet Verf. jedoch historisch gut belegt und detailliert die Entwicklungen nach, die letztlich zu der in den Reichsstrafgesetzen gefundenen und danach weiter entwickelten Aufgabenzuweisung an Staatsanwaltschaft und Gericht geführt haben. Treffend arbeitet er die Ungereimtheiten und Halbherzigkeiten heraus, die dadurch entstehen, dass die Staatsanwaltschaft als Zwitterwesen zwischen Auftrag der Durchsetzung staatlichen Strafrechts mit unvollständig ausgeprägter Unabhängigkeit und begrenzter Handlungsfähigkeit im Hauptverfahren ihre eigentliche Position nicht finden konnte. Demgegenüber sei die schon in der Literatur des 19. Jahrhunderts angemahnte Position als eine den Staat vertretende Partei im Strafverfahren ganz im Sinne des Zivilprozesses konsequent und ohne Systembrüche darzustellen.

Im 3. Kapitel wendet sich Verf. der Frage zu, ob denn die Inquisitionsmaxime des heutigen Verfahrensrechts nicht im Zusammenhang mit einem absoluten Strafbegriff stehe. Wie Verf. selbst erkennt, scheint eine solche Fragestellung vielleicht doch einer allzu fernen Vergangenheit anzugehören, um heute noch ausgiebig diskutiert werden zu müssen. Aber die weiteren Ausführungen ziehen den Leser dann doch so in ihren Bann, dass der zunächst vielleicht gedachte Vorwurf der Redundanz schnell korrigiert wird.

In Weiterentwicklung des mittelalterlichen Strafrechts, welches die Strafe eher als zweckrationale Reaktion verstand, änderte sich das Verständnis, den christlichen Vorstellungen folgend, in Richtung eines Theozentrierten Instruments zur Verwirklichung einer von Gott geforderten Gerechtigkeit. Sehr deutlich wurde dies etwa in Art. 69 der Carolina, wo letztlich zur Versöhnung des Täters mit Gott und der Gemeinschaft trotz hinreichender Beweismittel noch ein Geständnis des Beschuldigten gefordert wurde, weil nur durch die Einsicht in das verwirklichte Unrecht auch Reue 
und Vergebung möglich seien. Eine Vorschrift, die lange Zeit auch der Rechtfertigung von Folter diente.

Verf. bezweifelt aber, ob dies allein - wie Ignor und Seelmann bereits im Jahre 2002 vermuten - schon ausreiche, um die Schaffung des allein dem Richter überantworteten Inquisitionsprozess historisch nachzuweisen. Dies insbesondere unter Berücksichtigung der weiteren Entwicklungen des Strafverständnisses. Es folgt nun eine überaus fundierte Aufarbeitung der Geschichte der Theorien zum Strafbegriff, die von der Theozentriertheit zunächst in eine insbesondere von Pufendorf begründete weltliche, aus dem Nutzen der Gemeinschaft entwickelten Erklärung führte, die dann über Kants absolute Straftheorie in ihrer Fortentwicklung durch Hegel die Strafrechtsdiskussion des 19. und 20. Jahrhundert stark beeinflusst und sogar eine erneute Beschreibung der Strafe als göttliches Gebot (etwa Abegg und Ortloff) befördert habe. In den Diskussionen zur Reichsstrafprozessordnung ist denn auch immer wieder von der „heiligen Pflicht" des Staates zur Strafe und der „erhabenen Stellung “ die Rede, die das Strafrecht habe.

Letztlich belegt Verf. aufgrund seiner ebenso sorgfältigen wie umfänglichen historischen Analyse, dass nach dem Theozentrischen Strafverständnis des Mittelalters das gerade Ende des 18. Jahrhunderts erneute Erstarken der absoluten Straftheorien - gleichsam die metaphysische Überhöhung der Strafe ohne die Einbeziehung Gottes - notwendig dazu geführt habe, einen Parteiprozess im Strafverfahren abzulehnen, weil dieser allein die Zweckmäßigkeit des richterlichen Rechtsspruchs hätte rechtfertigen können, dem Anspruch der Verwirklichung einer idealisierten höheren Gerechtigkeit des Strafens jedoch nicht hinreichend gewachsen war.

Die Darlegungen sind historisch und theoretisch sehr gut recherchiert und wiedergegeben, bieten auch manch' neuen Zusammenhang, sind aber wohl in dieser Umfänglichkeit kaum erforderlich, um die Titelgebende Problematik zu diesem (Zwischen)Ergebnis zu führen, ist letzteres doch nur eine historische Erklärung für die Beibehaltung des - nach Ansicht des Verf's - systematisch und inhaltlich nicht haltbaren Inquisitionsprinzips.

Im 4. Kapitel wendet sich Verf. der Frage zu, ob die so entstandene noch heute gültige Prozessstruktur ein tragfähiges Fundament für den Strafprozess bildet. $\mathrm{Zu}$ diesem Zweck wendet er sich erneut den Straftheorien zu, dieses Mal aber, um ihre Bedeutung in der Gegenwart zu überprüfen und ihre Geeignetheit für ein modernes, rechtsstaatliches Strafverfahren zu untersuchen. Dass dies nach Ansicht des Verfassers schwerlich so sein kann hat er schon zuvor hinreichend deutlich gemacht und stellt dies auch gleich zu Anfang des Kapitels dar, indem er die Fragwürdigkeit der Vorstellung einer absoluten und auf göttlichem Willen und/oder moralischen Prinzipien beruhenden Strafgewalt des Staates aufzeigt; in einem modernen demokratischen 
Rechtsstaat sei vielmehr der einzige legitime Bezugspunkt für die Annahme und Ausübung staatlicher Gewalt der Souverän des Volkes, welcher den Staat in seiner konkreten Verfasstheit repräsentiere.

Nunmehr nimmt sich Verf. der aktuell vertretenen Straftheorien an, dekliniert sie durch und kritisiert sie - sehr zurecht - immer dann, wenn sie ins rein Sittliche oder gar Metaphysische abgleiten. Diese kritische Zusammenfassung hier im Detail zu wiederholen erscheint nicht sinnvoll. Nur einige Stichworte sollen gegeben werden, um den Gedankengang des Autors nachvollziehbar zu machen. So moniert er mit Kühl die Ungeklärtheit des Begriffs der Sozialethik; auch die weitgehend vertretene Auffassung, dass im Strafrecht rechtliche Schuld durch sittliche Schuld ergänzt werde; selbst wenn man mit Hirsch von einer reinen moralisch verstandenen Inhaltsbestimmung der Schuld Abstand nehme, hält Verf. dies für eine nicht legitime Beschreibung. Strafe müsse sich trotz ihrer Genugtuungsfunktion allein durch den rechtlichen Tadel zurechenbaren Verhaltens rechtfertigen. Hingegen seien Schuldausgleich und Prävention als Strafzwecke nicht von unterschiedlicher Art, sondern beschrieben lediglich zwei Seiten derselben Medaille. Wenn des Täters Schuld angemessen bestraft werde, so wirke dies für ihn wie auch für andere als negative Motivation (weitere) Straftaten zu begehen. Deshalb hält Verf. auch konsequent die Lehre von der positiven Sozialprävention lediglich für einen wichtigen empirischen Effekt der Strafe als Schuldausgleich, nicht aber für eine eigenständige Theorie.

Was die negative General- und Spezialprävention angeht, so kommt Verf. durch eine sehr eingehende Betrachtung der vertretenen Ansätze zu dem Ergebnis, dass trotz gewisser Probleme allein die Spielraumtheorie in der Lage ist, „, das Auseinanderfallen von präventiven und vergeltenden Elementen der Strafe zu verhindern und beide in der konkret zuerkannten Strafe zusammenzuführen. “ Letztendlich wendet sich Verf. damit von jedwedem auch nur metaphysisch angehauchten Begründungszusammenhang ab und beschreibt den Strafzweck als allein durch das berechtigte Genugtuungsinteresse der Allgemeinheit gerechtfertigt, wobei er bei letzterem mannigfaltige auch kriminalpolitische Zweckmäßigkeiten für legitim hält. Damit bestätigt Verf. seine vorangegangene Annahme von der republikanischen Natur der Strafgewalt. Das verstärkt er durch einem Vergleich mit dem Verwaltungsrecht, welches die staatliche Souveränität als von vornherein durch den Akt der Übertragung durch die Bürger begrenzt und dies durch verwaltungsrichterliche Kontrolle sowohl zu erkennen gibt als auch umsetzt. Folglich sei auch das Strafverfahrensrecht kein Schutzrecht für Individualrechte, sondern letztere seien lediglich potentielle Abwehransprüche, die jedoch nicht den Prozessgegenstand des gegenwärtigen Strafverfahrens bildeten. Auch diese Argumentation baut den Weg zum gut erkennbaren Ziel des Verf's weiter aus: dem Abbau hoheitlicher Vertreterstrukturen im Strafverfahren des modernen 
Inquisitionsprinzips hin zu einem dem Zivilprozessrecht eher entsprechenden Parteiund Verhandlungsprinzip.

In dem folgenden Teil des 5. und letzten Kapitels unterlegt Verf. seine Überlegungen verfassungstheoretisch und kommt damit zu seinem endgültigen Ergebnis, dass auch verfassungsrechtlich das vorliegende Strafverfahrensrecht inquisitorischer Prägung nicht zulässig, weil die Tätigkeit des Strafrichters nach diesem Modell keine wirkliche Rechtsprechende Tätigkeit sei. Vielmehr setze der Strafrichter den staatlichen Strafanspruch durch, statt als objektive Instanz die Bestrafung als Akt öffentlicher Gewalt objektiv zu überprüfen, wie dies bei den Verwaltungsgerichten im Rahmen der Bekämpfung staatlicher Akte individueller Belastung der Fall sei.

Diese in der Tat nach alledem nicht mehr sonderlich überraschende Erkenntnis erarbeitet sich Verf. durch eine überaus intensive Überprüfung von Inhalt und Ausmaß des verfassungsrechtlichen Begriffs der Rechtsprechung. Zunächst einmal stellt er klar, dass - anders als dies die Rechtsprechung des BVerfG's erscheinen lässt Art. 92 GG keine Definition dessen enthält, was Gegenstand der Rechtsprechung sein soll oder kann. Vielmehr ergebe sich aus der Vorschrift nur, dass die rechtsprechende Gewalt den Richtern anvertraut sei; was diese aber ausmache und was alles dieser Gewalt unterfalle ergebe sich nicht aus der Norm, hier böten spezielle Verfassungsvorschriften, wie etwa Art. 13 und 104 GG aber gewisse Hinweise. Letztendlich findet Verf. eine einleuchtende und gut begründete Definition dessen was Rechtsprechung sei, eine Definition, die zugleich die möglichen Gegenstände der rechtsprechenden Tätigkeit beschreibt: Die Entscheidung über das Bestehen eines Rechtsverhältnisses durch einen unbeteiligten Dritten. Eben dies leiste aber das Strafgericht nicht, soweit es als Sachwalter staatlicher Autorität zur Durchsetzung eines öffentlich rechtlichen Strafanspruchs auftrete.

Des Weiteren widerspreche der im Strafverfahrensrecht hoch aufgeladene Begriff der Waffengleichheit durchaus grundsätzlich der Architektur des geltenden Strafverfahrensrechts, da die Grundvoraussetzung dieser Annahme, die Gleichstellung von Bürger und Staat, gerade nicht gegeben sei - ein Umstand, der in der strafprozessualen Diskussion dadurch verdeckt wird, dass man versucht, die Waffengleichheit von Vornherein als begrenzt durch die strukturellen Vorgaben des Strafverfahrensrechts zu beschreiben. Auch die gerichtliche Untersuchungspflicht nach $\$ 244$ II StPO sei mit der Rolle des objektiven Entscheiders über ein zwischen Staat und Bürger strittiges Rechtsverhältnis - der Straftat - nicht vereinbar und stehe überdies mit der akzeptierten und nunmehr sogar legalisierten Absprachepraxis in Widerspruch.

Die Ausführungen sind wohlbegründet und durchaus schlüssig, wenngleich sie letztlich zu dem Verdikt der Verfassungswidrigkeit des gegenwärtigen Strafverfahrensrechts in Bezug auf die richterliche Tätigkeit führen. Ebenfalls muss anerkannt wer- 
den, dass die vom Verf. angedeutete Umstrukturierung des Strafverfahrens in einen hoheitlich exekutiven Teil, der durch die Staatsanwaltschaft vertreten wird und einen wirklichen gerichtlichen Teil, in welchem die (realen oder beantragten) Belastungen des Bürgers durch die Exekutive unabhängig überprüft werden, eine Vielzahl heute bestehender Widersprüche zum Wegfall zu bringen vermag: etwa die Vorbelastung des Gerichts durch den Eröffnungsbeschluss, die Gewährung einer wirklichen Waffengleichheit, die Einpassung von unterschiedlichsten Praktiken der Abrede bzw. Verfügung über den Verfahrensgegenstand in das Verfahrensrecht und vieles mehr. Auch die Schutzwirkung des Gerichtsverfahrens zugunsten des Bürgers könnte sich reiner entfalten, weil das Gericht nicht mehr staatliche Interessen der Strafverfolgung zu vertreten hätte, sondern diese von der Staatsanwaltschaft einzufordernden Interessen objektiv gegenüber dem betroffenen Bürger zu überprüfen hätte.

Ob letztlich eine solche Neustrukturierung des deutschen Strafverfahrens auch praxistauglich wäre, wie sie im Detail auszusehen hätte und wie weit die bekannten negativen Implikationen des anglo-amerikanischen Parteiverfahrens dadurch gleichsam „mit eingekauft“ wären, dies alles bedarf, worauf der Autor ausdrücklich hinweist, der weiteren Überprüfung und Erforschung. Sicherlich aber hat die Studie von Haas einen überaus wichtigen Beitrag zur Diskussion um wesentliche Strukturen des deutschen Strafverfahrensrechts geleistet, der künftig zu beachten ist und durch seine umfängliche Dokumentation auch gut als Nachschlagewerk zu nutzen ist.

Nach dieser sehr positiven Bewertung sollen aber kleinere Monita nicht ganz verschwiegen werden. Die Fülle der vorgelegten Einzelheiten und ihrer Diskussion steht in Gefahr, auch den wohlmeinenden Leser zu erschlagen und von dem roten Faden der Studie abzulenken. Sicherlich ist es nicht uninteressant, noch einmal eine Reise durch die mit der Strafzweckproblematik eng verbundenen traditionellen Grundfragen zu machen, wobei so manche Lücke gefüllt werden mag. Auf der anderen Seite erscheint jedoch dieser Aufwand für die Bearbeitung in Hinblick auf die Themengebende Fragestellung mitunter als allzu groß und für die Zielführung der Argumentation bei aller gedanklichen Tiefe nicht wirklich in dieser Form erforderlich zu sein. Der Leser versinkt allzu schnell in einer Fülle von Einzelfragen, deren unmittelbare Relevanz für den Fortgang der Arbeit nicht leicht zu erkennen ist. Freilich muss diese kritische Anmerkung ein wenig abgemildert werden, da es sich um eine Habilitation handelt, bei der bekanntlich eher der psychologische und mitunter auch der reale Zwang besteht mehr wissenschaftliche Handwerkskunst zu zeigen, als das Thema dies eigentlich erfordert.

Und noch ein Letztes: es geht in der Studie auch nicht um die Prozessstruktur als Ganze, wie am Anfang in Aussicht gestellt, sondern nur um die Struktur soweit Staatsanwaltschaft und Gericht betroffen sind. Die gleich zu Beginn offen gelegte Ausklammerung der Verteidigung aus den Betrachtungen macht das unmöglich. So 
haben wir es - etwas anders als der Autor vermuten lässt - mit einer Studie über die widersprüchliche oder gar verfassungswidrige Position des Gerichts im Rahmen des modernen, rechtsstaatlich geläuterten Inquisitionsprozess deutschen Rechts zu tun. Als solche ist sie jedoch hervorragend und hat die strafprozessuale Grundsatzdiskussion deutlich bereichert. 